\title{
A influência do estado nutricional durante o período gestacional e sua correlação no peso do recém-nascido
}

The influence of the nutritional state during the gestacional period and its correlation in the weight of the newborn

La influencia del estado nutricional durante el período gestacional y su correlación en el peso del recién nacido

Hidelânia Batista Monteiro de Freitas ${ }^{1}$, Rafael Ferreira Lima ${ }^{2}$, Mayra Vieira Pereira Targino ${ }^{1}$, Ayssa Letícia Vieira Pereira Targino ${ }^{1}$, Ailton Targino do Nascimento ${ }^{1}$, Larruama Priscylla Fernandes de Vasconcelos $^{1 *}$, Débora Gomes de Sousa Araújo', Walnara Arnaud Moura Formiga1, Jobyson Gervázio Soares ${ }^{3}$, Íngrid Raquel Martins Gomes Fernandes ${ }^{1}$, Dárcio Romário de Lucena Calisto ${ }^{1}$, Thiago Alves Viana ${ }^{1}$.

\section{RESUMO}

Objetivo: Investigar a influência do estado nutricional durante o período gestacional correlacionado ao peso do recém-nascido. Métodos: A presente pesquisa possuiu caráter exploratório descritivo com abordagem quantitativa. O estudo foi realizado na Unidade Básica de Saúde (UBS) no Município de São Bento - PB. A pesquisa foi realizada com 24 prontuários de gestantes atendidas na UBS, não sendo necessário utilizar a totalidade dos prontuários. Resultados: Foi evidenciado o IMC da primeira consulta do pré-natal com 12,5\% das gestantes em estado de desnutrição, $37,5 \%$ em eutrofia e $50 \%$ com excesso de peso e obesidade. O IMC relacionado ao final da gestação, foi verificado uma progressão de $75 \%$ para o excesso de peso e obesidade. Quanto ao peso dos recém-nascidos foi observado, que 29,2\% apresentavam peso insatisfatório e baixo peso, com predominância de peso adequado $70,8 \%$. Conclusão: Diante dos resultados, esse fato mostrou a importância do profissional de nutrição durante o acompanhamento pré-natal, para uma gestação mais saudável tanto para a mãe como para o bebê, visando um parto mais seguro em relação a complicações.

Palavras-chave: Gestante, Estado nutricional, Recém-nascido, Pré-natal.

\footnotetext{
ABSTRACT

${ }^{1}$ Faculdades Integradas de Patos - FIP; *E-mail: larruama priscylla@hotmail.com

2 Universidade Federal de Campina Grande - UFCG;

${ }^{3}$ Centro Universitário João.
}

Objective: To investigate the influence of nutritional status during the gestational period correlated with the weight of the newborn. Methods: The present research had descriptive exploratory character with quantitative approach. The study was conducted at the Basic Health Unit (UBS) in the Municipality of São Bento - PB. The study was carried out with 24 charts of pregnant women attended at UBS, and it was not necessary to use all the medical records. Results: The BMI of the first prenatal visit was evidenced with $12.5 \%$ of the pregnant women in malnutrition status, $37.5 \%$ in eutrophy and $50 \%$ in overweight and obesity. The BMI related to the end of gestation, a progression of $75 \%$ for excess weight and obesity was verified. The weight of the newborns 
was observed, that $29.2 \%$ had unsatisfactory weight and low weight, with a predominance of adequate weight $70.8 \%$. Conclusion: In view of the results, this fact showed the importance of the nutrition professional during the prenatal care, for a healthier pregnancy for both the mother and the baby, aiming at safer delivery in relation to complications.

Keywords: Pregnant, Nutritional status, Newborn, Prenatal.

\section{RESUMEN}

Objetivo: Investigar la influencia del estado nutricional durante el período gestacional correlacionado con el peso del recién nacido. Métodos: La presente investigación poseyó carácter exploratorio descriptivo con abordaje cuantitativo. El estudio fue realizado en la Unidad Básica de Salud (UBS) en el Municipio de São Bento - PB. La investigación fue realizada con 24 prontuarios de gestantes atendidas en la UBS, no siendo necesario utilizar la totalidad de los prontuarios. Resultados: Se evidenció el IMC de la primera consulta del prenatal con el $12,5 \%$ de las gestantes en estado de desnutrición, el $37,5 \%$ en eutrofia y el $50 \%$ con sobrepeso y obesidad. EI IMC relacionado al final de la gestación, se verificó una progresión del $75 \%$ para el exceso de peso y obesidad. En cuanto al peso de los recién nacidos fue observado, que el $29,2 \%$ presentaba peso insatisfactorio y bajo peso, con predominancia de peso adecuado 70,8\%. Conclusión: Ante los resultados, este hecho mostró la importancia del profesional de nutrición durante el seguimiento prenatal, para una gestación más saludable tanto para la madre y para el bebé, buscando un parto más seguro en relación a complicaciones.

Palabras clave: Gestante, Estado nutricional, Recién nacido, Prenatal.

\section{INTRODUÇÃO}

Discute-se muito atualmente sobre a importância do estado nutricional da mãe para o desenvolvimento do bebê. É evidente em diversos estudos a afirmação que, carências nutricionais da mulher podem provocar não só o crescimento intrauterino deficiente, como muitas outras alterações, especialmente no que diz respeito ao sistema nervoso central do recém-nascido (BRUNO et al., 2009; ALMEIDA, 2007).

A inadequação do estado antropométrico materno, tanto pré-gestacional quando gestacional, constitui-se um problema de saúde pública, tendo em vista que favorece o desenvolvimento de intercorrências gestacionais e influencia as condições de saúde do feto a saúde materna no pós-parto (PADILHA et al., 2007).

A gravidez é um período que impõe necessidades nutricionais aumentadas, e a adequada nutrição é indispensável para a saúde da mãe e do bebê. Durante a gestação, há necessidade adicional de energia por causa do crescimento do feto, da placenta, dos tecidos maternos, bem como para o próprio consumo da gestante (AFONSO e SONATI, 2011).

É crescente a preocupação com a nutrição e o crescimento desde a vida intrauterina, visando evitar as deficiências. Nesse contexto, a assistência pré-natal deve propiciar o diagnóstico nutricional precoce das gestantes e se necessário promover a recuperação nutricional, bem como garantir o adequado ganho de peso na gravidez, a fim de evitar os desvios do crescimento fetal (FUJIMORE, 2011).

O suporte nutricional adequado durante o período gestacional é decisivo para o curso gestacional. A dieta, no primeiro trimestre da gestação, é muito importante para o desenvolvimento e diferenciação dos diversos órgãos fetais. Já nos trimestres subsequentes, a dieta está mais envolvida com a otimização do crescimento e do desenvolvimento cerebral do feto (DREHMER et al., 2008).

Além de interferir na qualidade da gravidez e no peso ao nascer as deficiências nutricionais são responsáveis por grande parte da morbidade e mortalidade perinatais. Se a ingestão dietética for insuficiente e se os estoques de nutrientes da mãe estiverem baixos, o feto precisará recorrer às reservas préconcepcionais para se suprir, ocasionando comprometimento do binômio materno-fetal (FUJIMORE, 2011). Assim, de uma maneira geral, a diminuição da ingestão de nutrientes pode provocar alterações nos mecanismos maternos de adaptação à gravidez e diminuir o transporte de nutrientes (BRUNO et al., 2009). 
Dessa forma, vale interrogar se o peso da criança, ao nascer, reflete a qualidade da atenção dispensada à gestante, seu estado nutricional antes e durante a gestação e os fatores de risco aos quais está exposta?

Mediante discussão acerca da relação entre o estado nutricional da gestante e o peso de seus recém nascidos, este estudo investigou se as mulheres com uma nutrição adequada durante a gravidez têm menos complicações e dão à luz bebês maiores e mais saudáveis, se há relação direta entre o peso de nascimento e o ganho de peso materno durante a gestação e por fim se a diminuição da ingestão de nutrientes pode provocar alterações nos mecanismos maternos de adaptação à gravidez e diminuir o transporte de nutrientes adequado.

A cada dia surgem mais discussões a respeito desse momento tão importante para a vida da maioria das mulheres, exigindo-se cada vez mais conhecimentos e experiências que venham enriquecer e melhorar a qualidade de vida tanto da gestante quanto do bebê que vai nascer.

Diante do exposto, a presente pesquisa teve como objetivo geral investigar a influência do estado nutricional durante o período gestacional e sua correlação com peso do recém-nascido. Além de analisar os fatores socioeconômicos, investigar através dos prontuários os fatores de riscos que estão relacionados ao baixo peso do recém-nascido, contribuir junto a UBS com a divulgação dos resultados visando reduzir tal problema.

\section{METODOLOGIA}

O presente estudo possuiu caráter exploratório descritivo com abordagem quantitativa, realizado na Unidade Básica de Saúde (UBS) no Município de São Bento - PB. A pesquisa exploratória visa proporcionar uma visão geral de tipo aproximativo, acerca de um determinado fato. Constitui a primeira etapa de uma investigação mais ampla, formulando hipóteses e tornando o problema mais explícito (GIL, 2009). O método de pesquisa descritivo tem como característica observar, registrar, analisar, classificar e correlacionar fatos, sem que o pesquisador interfira neles, procurando descobrir a frequência com que o fenômeno acontece e sua relação com outros fatores (ANDRADE, 2009).

A população do presente estudo foi composta por coleta de análise de 24 prontuários de pacientes que já tiveram atendimento realizado na UBS Benedito Pedro da Silva, localizada no município de São Bento - PB.

Quantos aos critérios de inclusão, foram selecionados prontuários das gestantes que foram atendidas na UBS e que fizeram o acompanhamento do pré-natal regularmente. Já para os critérios de exclusão estes incluíram os prontuários que não estavam devidamente cadastrados e não tenha ocorrido o registro do acompanhamento regular do pré-natal.

O instrumento de pesquisa utilizado para investigar a influência do estado nutricional durante o período gestacional correlacionando ao peso do recém-nascido, em gestantes atendidas na UBS em São Bento -PB, foi realizado através de um questionário previamente elaborado, com questões estruturadas com base no modelo do prontuário da gestante, envolvendo fatores sócio-demográficos e questionamentos sobre a saúde materna.

Os prontuários foram repassados para o pesquisador conforme a organização do serviço da unidade. Foi realizada a coleta dos prontuários referentes aos meses de novembro de 2016 a março de 2017. Os dados foram coletados durante o mês de fevereiro a março de 2017. A pesquisa foi possível a partir da assinatura do Termo de Risco, Sigilo e Confidencialidade.

Os dados coletados pela pesquisa foram computados e analisados a partir de planilhas do Excel e programa estatístico Statistical Package For The Social Sciences (SPSS) versão 22.0.0.0 para Windows. Foi 
utilizada a estatística descritiva utilizando a frequência, média e desvio padrão, com nível de significância para $p<0,05$. Posteriormente os dados foram apresentados em tabelas ou gráficos para melhor compreensão dos resultados.

Este estudo foi submetido à aprovação do Comitê de Ética e Pesquisa (CEP) envolvendo seres humanos das Faculdades Integradas de Patos - FIP. A presente pesquisa teve início após a aprovação pelo CEP das FIP com o parecer n. 1.983.877, com o Termo de Autorização Institucional, devidamente assinado pelo Secretário de Saúde do Município de São Bento - PB, também, responsável pela UBS.

A pesquisa obedeceu aos critérios da Resolução no 466/12 aprovada pelo Conselho Nacional de Saúde Ministério da Saúde (CNS - MS) (BRASIL, 2012), que trata de pesquisas envolvendo seres humanos direta ou indiretamente, assegurando a garantia de privacidade e anonimato dos dados.

Foi entregue também, junto aos CEP das FIP (responsável pela Instituição), o Termo de Compromisso o Pesquisador, que declaram a responsabilidade do cumprimento das normas vigentes.

\section{RESULTADOS E DISCUSSÃO}

A pesquisa contou com a participação de 24 prontuários de gestantes que já foram atendidas na UBS Benedito Pedro da Silva, os dados foram coletados e os resultados foram analisados por meio do SPSS versão 22.

A figura 1, mostra a prevalência de antecedentes familiares com Doenças Crônicas Não Transmissíveis (DCNT) nas gestantes estudadas. Os resultados encontrados mostram que o número de gestantes com antecedentes de DCNT é de 37,7\% para hipertensão, 33,3\% possuíam diabetes, e apenas $29 \%$ delas não tinha nenhum antecedente de DCNT. É importante que seja realizado o acompanhamento do pré-natal em virtude dos problemas encontrados na família.

Figura 1. Antecedentes Familiares de Doenças Crônicas Não Transmissíveis. São Bento-PB, 2017.

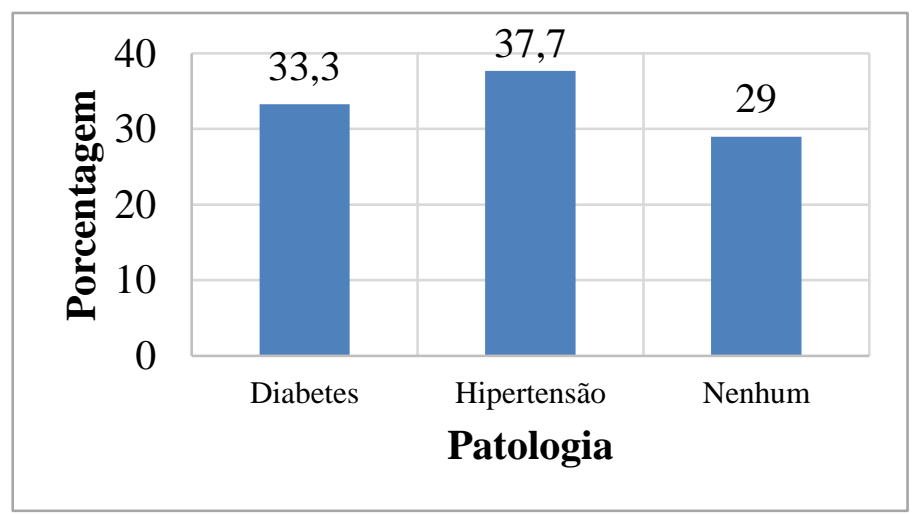

Fonte: Dados da própria pesquisa.

Xavier et al. (2013), investigando o risco reprodutivo e renda familiar de gestantes, encontraram resultados, onde $33,0 \%$ das gestantes apresentavam hipertensão e $22 \%$ com relatos de diabetes na família.

De acordo com Ribeiro et al. (2015), a prevalência de Diabetes Mellitus (DM) tem aumentado progressivamente, mais 382 milhões de pessoas em todo o mundo têm diabetes, o que corresponde a 8,3\% 
da população. O Diabetes Gestacional (DG) é definido como qualquer grau de intolerância à glicose com início ou detecção durante a gravidez. O DG é um fator de risco para o DM.

Segundo Packer (2016), no Brasil cerca de 7\% das gestações são complicadas pela hiperglicemia. A gestação é caracterizada por um acréscimo na resistência periférica à insulina e por incremento na produção de insulina pelas células-beta do pâncreas. A resistência à insulina aumenta durante a gestação em virtude da secreção placentária de alguns hormônios considerados diabetogênicos, como hormônio do crescimento, cortisol e hormônio lactogênico placentário. O metabolismo energético pode ser dividido em duas fases distintas: a primeira fase é conhecida como anabólica, na qual a glicemia das gestantes tente a diminuir, em especial quando ocorre jejum prolongado. A segunda fase é a catabólica, com crescente consumo de nutrientes maternos pelo feto. Nela, torna-se evidente o aumento da resistência periférica à insulina.

Para Brasil (2010) e Costa et al. (2010) a Hipertensão Arterial (HA) quando atinge gestantes, há risco para a saúde tanto da mãe quanto do bebê e, se a gestação não for vivenciada de forma saudável, então é considerada "gestação de alto risco", a qual é definida como uma situação limítrofe que pode implicar riscos tanto para a mãe quanto para o feto e há um determinado número de gestantes que, por características particulares, apresentam maior probabilidade de evolução desfavorável.

A figura 2 expressa as doenças adquiridas durante a gestação. Pode-se observar que a maioria das gestantes atendidas na unidade mostraram-se livre de adquirir patologias durante a gestação (66,7\%). Seguindo a análise, 25\% tiveram infecção urinária durante a gestação e apenas 8,3\% tiveram alterações na pressão arterial.

Figura 2. Patologias adquiridas na gestação. São Bento-PB, 2017.

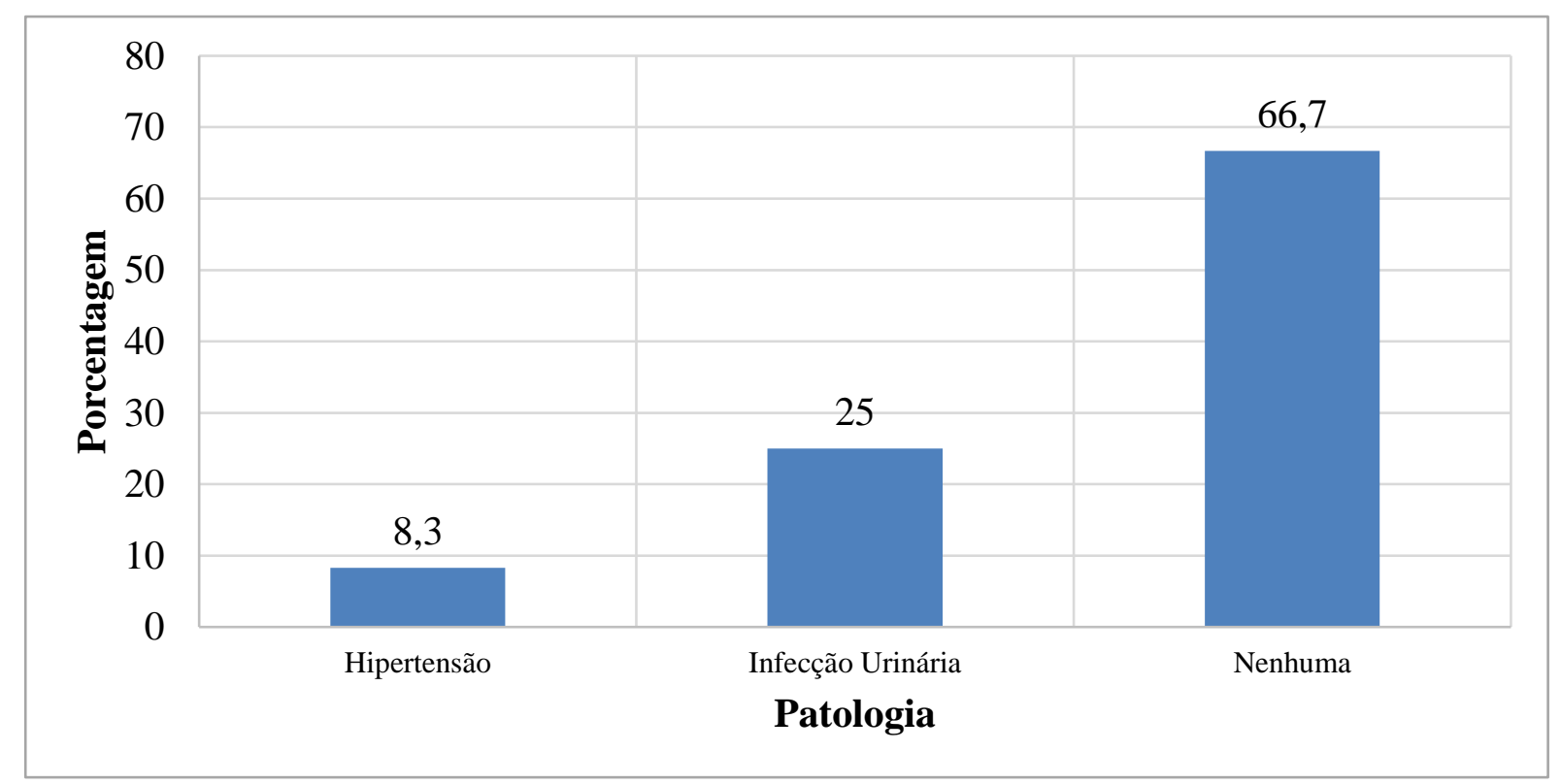

Fonte: Dados da própria pesquisa.

Segundo Moura et al. (2011) e Soares et al. (2009) estes abordam que a hipertensão na gestação é a maior causa de morte materna no país, atingindo cerca de 35\% dos óbitos a partir de uma taxa de 140-160 mortes maternas $/ 100.000$ dos nascidos vivos. Evitável em mais de $90 \%$ dos casos, a mortalidade materna configura-se como uma importante violação dos direitos reprodutivos. Além de apontar a qualidade na atenção à saúde que está sendo prestada, é considerada um dos indicadores da assistência à saúde das mulheres. 
Duarte et al. (2008) e Darzé et al. (2011) descrevem que as alterações anatômicas e fisiológicas que ocorrem no sistema urinário durante a gravidez facilitam ainda mais o desenvolvimento de infecções urinárias sintomáticas em mulheres que já apresentaram bacteriúria anteriormente. Essas condições podem levar ao agravamento, tanto para o prognóstico materno quanto para o prognóstico perinatal. Entre as complicações, destacam-se o trabalho de parto prematuro, ruptura prematura de membrana amnióticas, restrição de crescimento intra-úterino, recém-nascidos de baixo peso e óbito perinatal.

Ramos et al. (2016), avaliando 432 gestantes com idade de 17 a 41 anos, encontraram prevalência de infecção urinária em $25,46 \%$ delas em pelo menos um dos três semestres de gestação, $5,32 \%$ apresentaram em dois semestres e $0,93 \%$ apresentaram em todos os semestres de gestação.

A figura 3 expressa as informações do Índice de Massa Corporal (IMC) das gestantes, aferida na primeira consulta do pré-natal. Foi observado que as gestantes se encontravam em um estágio de excesso de peso $(33,3 \%)$ e obesidade $(16,7 \%)$ o que totaliza $(50 \%)$, fator preocupante, quando comparado com a amostra analisada. Foi identificado que $12,5 \%$ das gestantes encontravam-se em estado de desnutrição e $37, \%$ em eutrofia. Ainda na figura 3 é possível observar as informações referentes ao IMC final das gestantes atendidas no pré-natal. Foi identificado que as mesmas elevaram os valores para obesidade $(45,9 \%)$, fator extremamente preocupante, em virtude dos fatores de risco associados a gestação totalizando excesso de peso e obesidade $(75 \%)$ da amostra analisada.

Figura 3. Decorrendo do IMC das gestantes estudada, inicial e final, durante o período gestacional. São Bento-PB, 2017.
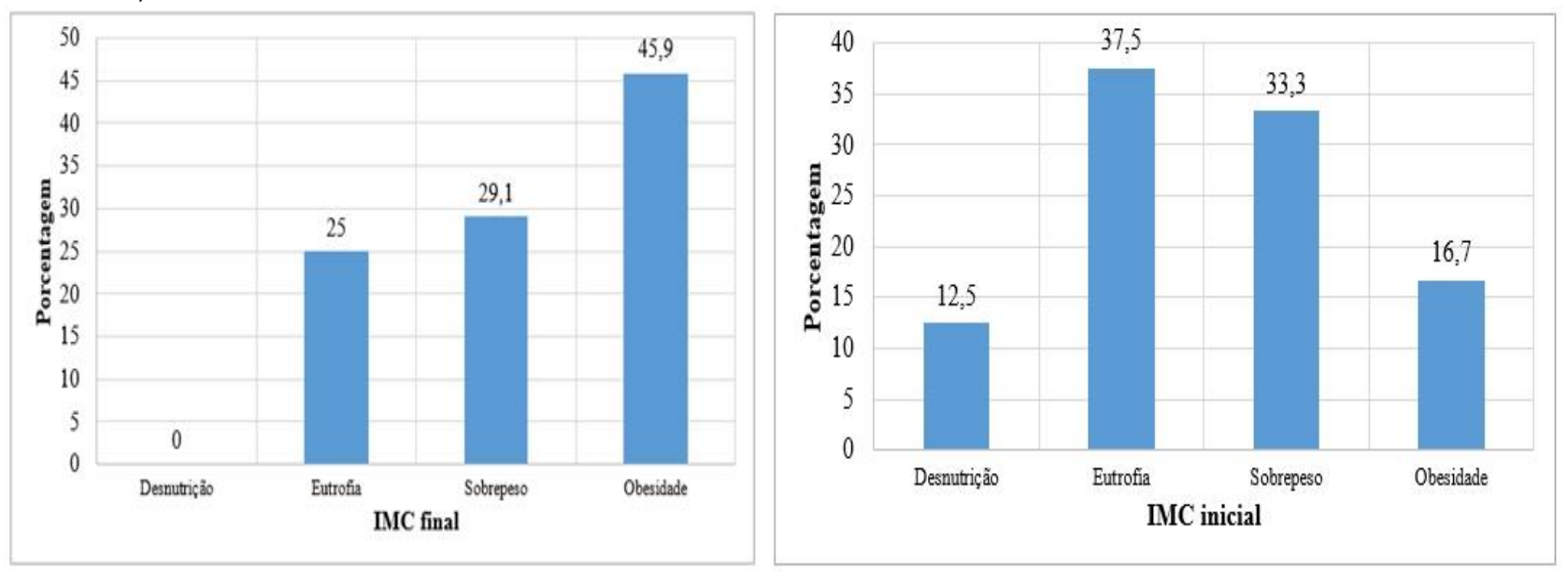

Fonte: Dados da própria pesquisa.

Para Mendonça e Reticena (2012) a avaliação do estado nutricional de gestantes deve ser realizada continuamente ao longo desse período. Esse acompanhamento da mulher durante a assistência pré-natal tem como objetivo estabelecer o estado nutricional, identificar fatores de risco, possibilitar interferências terapêuticas e profiláticas no sentido de corrigir distorções e planejar a educação nutricional.

Para a OMS (2003), os aspectos antropométricos maternos e o consumo adequado de nutrientes são os maiores determinantes do crescimento fetal, com repercussões no peso e a idade gestacional ao nascer. A identificação precoce do inadequado estado nutricional das gestantes colabora para intervenções oportuna resultando em impactos positivos nas condições do nascimento da criança e minimizando as taxas de mortalidade perinatal e neonatal. 
Santos et al. (2014), encontrou resultados semelhantes com o do presente estudo, onde a prevalência de IMC classificado como obesidade foi de $37,5 \%$, seguido de uma diferença significante dos IMC's classificados como sobrepeso e eutrofia, apresentaram respectivamente, 29,2\% e 20,8\%; com menor prevalência, estavam as gestantes classificada como baixo peso, apresentando $12,5 \%$.

Para Barros e Nicolau (2014), o IMC gestacional pode ser utilizado para detectar o risco de um prognóstico adverso na gestação, e assim planejar intervenções nutricionais para diminuir ou eliminar complicações na saúde materno-infantil.

Stulbach et al. (2007), relata que o ganho ponderal excessivo durante a gestação como uma possível causa da obesidade entre mulheres. Este aumento está associado a algumas complicações, entre elas, macrossomia fetal, as hemorragias, o trauma fetal, baixo peso ao nascer e mortalidade infantil. Ainda a obesidade na gravidez apresenta um risco maior de parto muito antes do termo (menos de 32 semanas), além de morte precoce ou tardia fetal se esta for sua primeira gravidez.

A figura 4, mostra o peso ao nascer dos lactentes das gestantes atendida na unidade. A maioria dos lactentes apresentaram peso adequado ao nascer $(70,8 \%)$, sendo esse um indicador de qualidade do estado nutricional. Com uma diferença significantes vem os nascidos que apresentam peso insuficiente ao nascer $(20,9 \%)$ e com menor prevalência aqueles que nasceram com baixo peso ao nascer $(8,3 \%)$.

Figura 4. Peso ao nascer. São Bento-PB, 2017.

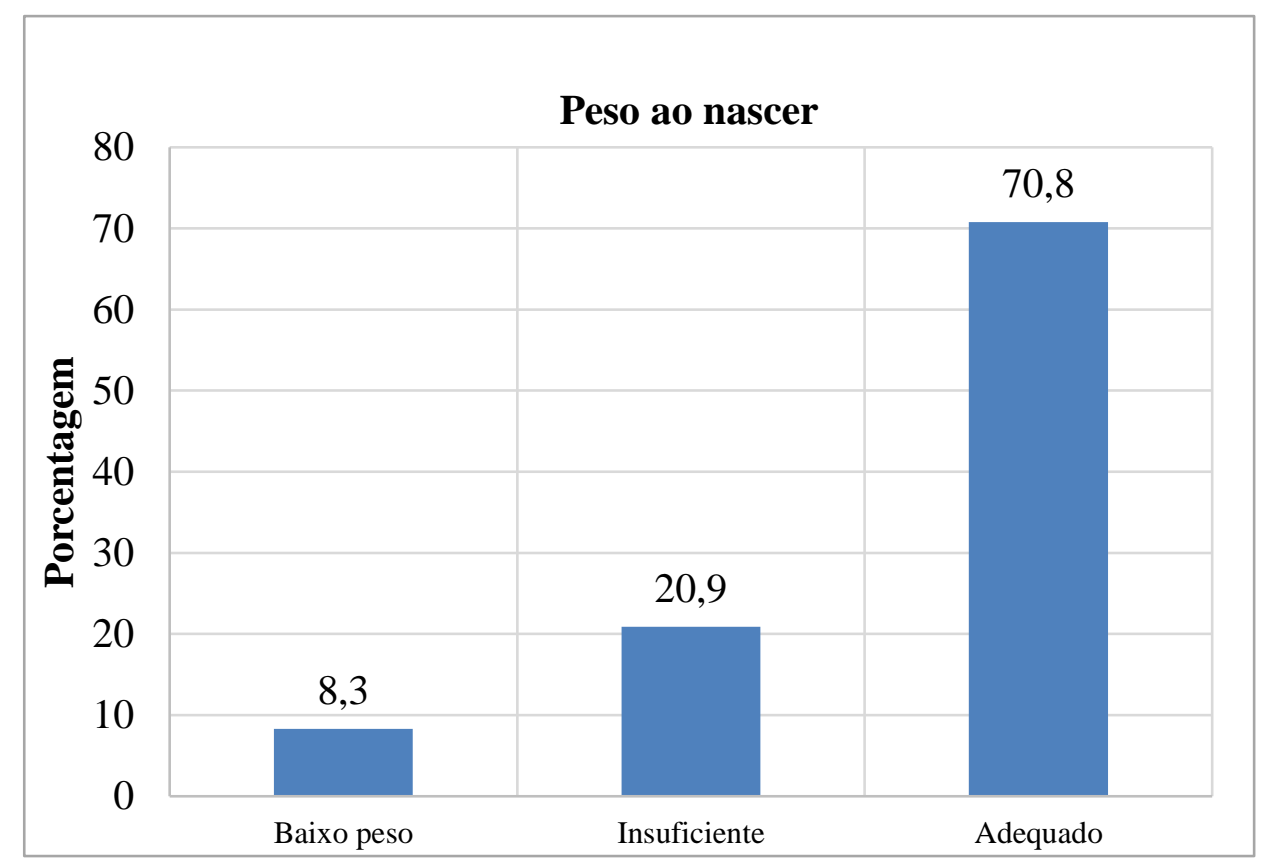

Fonte: Dados da própria pesquisa.

Resultados semelhantes foram encontrados por Sousa et al. (2015), quando os lactentes apresentaram, prevalência de $88,9 \%$ para os nascidos com peso adequado, $6,7 \%$ apresentam peso insuficiente, e $4,4 \%$ baixo peso ao nascer.

De acordo com Dietz et al. (2009), o baixo peso ao nascer e a prematuridade são reconhecidos como fatores de risco para a mortalidade neonatal. Entretanto, é importante considerar que os dois extremos de 
peso fetal constituem fatores de risco e de agravos à saúde da criança, destacando-se os baixos índices de Apgar.

A figura 5 exibe o número de consultas que as gestantes participaram ao longo da gestação, foi observado que $54 \%$ das gestantes obtiveram um total de 8 consultas no decorrer do pré-natal. Em seguida de $16 \%$ para 6 consultas e $30 \%$ para 7 atendimentos.

Figura 5. Número de consultas durante o pré-natal. São Bento-PB, 2017.

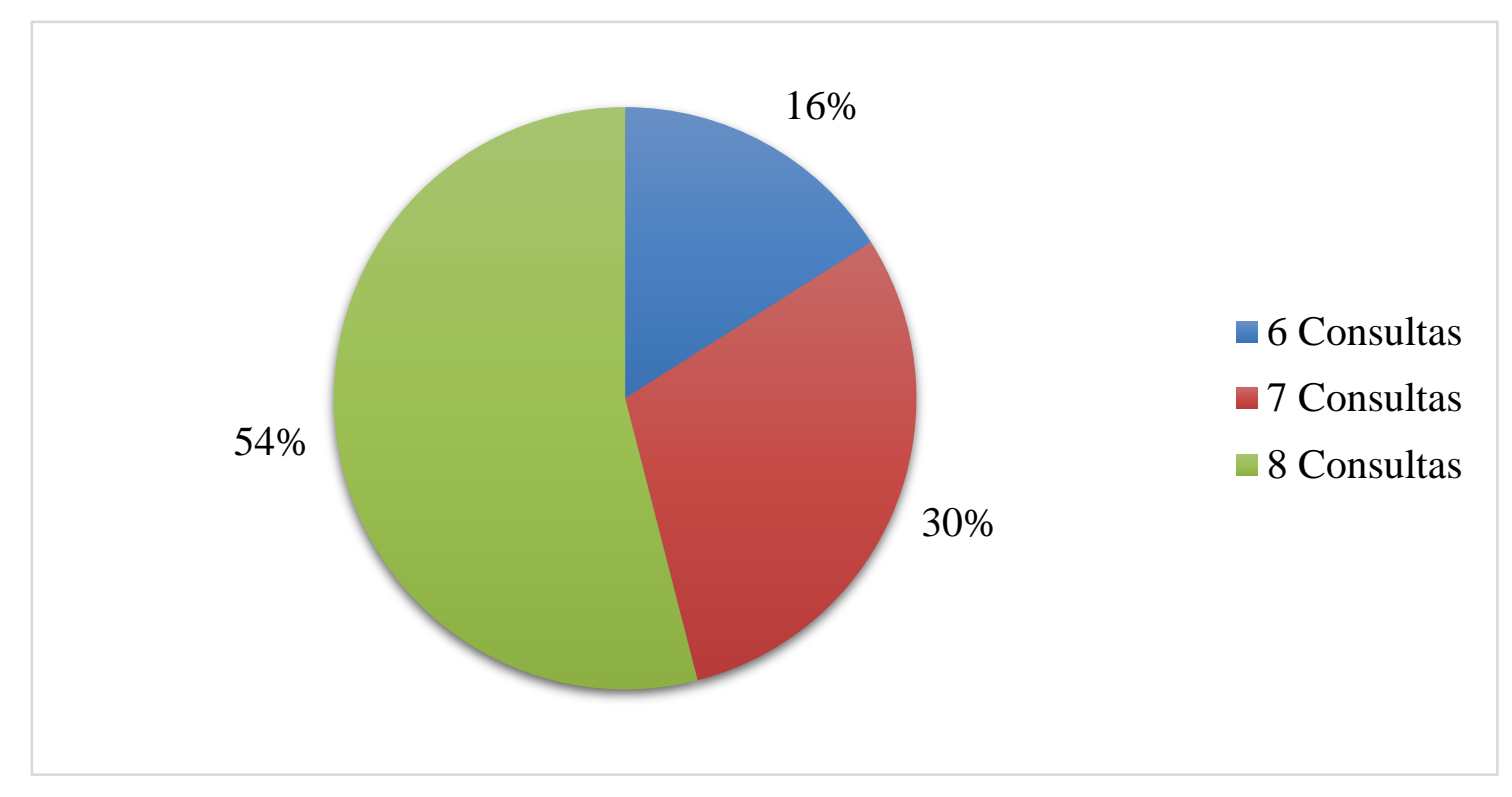

Fonte: Dados da própria pesquisa.

Segundo Almeida e Barros (2005) e Junior et al. (2014), o Ministério da Saúde preconiza no mínimo seis consultas pré-natais, sendo a primeira no primeiro trimestre, outras duas no segundo trimestre e as três últimas no terceiro trimestre de gestação. Acrescenta também, que a primeira consulta seja até 17 semanas de gestação. Além do número de consultas, ressalta-se que as mesmas sejam de boa qualidade, aspecto que muitas vezes é negligenciado.

Libera et al. (2014), relatam que a eficácia da assistência nutricional durante o pré-natal têm sido tema decorrentes em estudos que confirmam os benefícios da nutrição adequada sobre o resultado da gestação. Completa dizendo, que no Brasil, o profissional nutricionista, no âmbito da atenção básica, tem o compromisso de atuar, de forma humanizada, integrada e planejada no desenvolvimento de ações de alimentação e nutrição, bem como avaliar a implementação das ações, a medida de seu impacto sobre a situação de saúde da população e participar para contribuição de atividades de Educação Permanente.

Diante do exposto, é notório a essencialidade do profissional nutricionista na assistência pré-natal para melhor acompanhar a gestação para uma gravidez saudável, assim como o momento do parto diminuir as complicações existentes, no momento do parto e ao longo da vida da criança.

\section{CONCLUSÃO}

A partir dos resultados da pesquisa foi possível concluir que o inicio do pré-natal as gestantes foram classificadas como excesso de peso e obesidade com progressão até o final da gestação, o que justifica as 
gestantes também desenvolverem a hipertensão, a qual também pode-se associar com o peso elevado. Quando se tratou do número de consultas, foi evidente que todas as gestantes realizaram um número considerável de consultas, sendo que não ocorreu envolvimento do profissional nutricionista associado ao pré-natal, sendo isso uma explicação para o ganho de peso durante a gestação. Os discretos casos de peso insuficiente e baixo peso, provavelmente em virtude de uma nutrição inadequada durante a gestação, o que pode ter causado esse peso reduzido. Este fato mostra a importância do profissional de nutrição durante o acompanhamento pré-natal, para uma gestação mais saudável, tanto para a mãe como para o bebê, assim como também um parto mais seguro em relação a complicações.

\section{REFERÊNCIAS}

1. AFFONSO CV, SONATI JG. Nutrição e o Ciclo da Vida: Gravidez, Amamentação e a Criança Pré-Escolar. São Paulo: Editora e Consultoria em Nutrição Ltda, 2011.

2. ALMEIDA MF. Risk-factors for antepartum fetal deaths in the city of Sao Paulo, Brazil. Revista de Saúde Pública, 2007; 41(1):35-43.

3. ALMEIDA SDM, BARROS MBA. Equidade e atenção à saúde de gestantes em Campinas (SP). Revista Panamericana de saúde Pública, 2005; 18(1): 15-25.

4. ANDRADE MM. Introdução à metodologia do trabalho científico: elaboração de trabalhos na graduação. São Paulo: Atlas, 2009.

5. BRASIL. Ministério da Saúde. Secretaria de Atenção à Saúde, Departamento de Ações Programáticas Estratégicas. Gestação de Alto risco: Manual Técnico. 5 ed. Brasília: Ministério da Saúde, 2010.

6. BRASIL. Conselho Nacional de Saúde. Resolução №466, de 2012. Pesquisa Envolvendo Seres Humanos, Brasília, DF, jun. 2013.

7. BARROS MAR, NICOLAU AI. O. Fatores nutricionais e maternos e repercussão no peso do recém-nascido. Revista de Enfermagem da UFPI, 2014; 3(2): 49-55.

8. BRUNO, IR; FELIX, RC; SALADO, GA. Relação da condição socioeconômica de gestantes e seus hábitos alimentares e possível influencia no peso ao nascer. Encontro Internacional de Produção Científica Centro Universitário de Maringá, 2009. p. 63.

9. COSTA RS. Fatores associados ao peso de nascimento insuficiente no Hospital Regional de Cotia. Dissertação do Mestrado em Saúde Pública, Universidade São Paulo, São Paulo - SP, 2010.

10. DREHMER M, CAMEY S, SCHMIDT MI et al. Socioeconomic, demographic and nutritional factors associated with maternal weight gain in general practices in Southern Brazil. Cadernos de Saúde Pública, 2010; 16(5): 1024-1034.

11. DARZÉ OISP, BARROSO U, LORDELO M. Preditores clínicos de bacteriúria assintomática na gestação. Revista Brasileira de Ginecologia e Obstetrícia, 2011; 33(8):196-200.

12. DIETZ PM, CALLAGHAN WM; SMITH R et al. Baixo ganho de peso na gestação e pequeno para idade gestacional: uma comparação da associação com três diferentes medidas de pequeno porte para a idade gestacional. Jornal Americano de Obstetrícia e Ginecologia, 2009; 201(1): 1-7.

13. DUARTE G, MARCOLIN AC, QUINTANA SM et al. Infecção urinária na gravidez. Revista Brasileira de Ginecologia e Obstetrícia, 2008; 30(2):93-100.

14. FUJIMORE E. Evolucion del estado nutricional de embarazadas atendidas em la red básica de salud. Santo Andre, Brasil. Revista Latino-americana de Enfermagem, 2011; 9(3):64-69.

15. GIL AC. Métodos e técnicas de pesquisa social. São Paulo: Atlas, 2009. p. 49

16. MEDEIROS JÚNIOR A, SILVA AMDF, LOPES LFL. Qualidade pré-natal em relação às sorologias sífilis, HIV e hepatite $B$ em gestantes de uma unidade de saúde de Natal - RN. Revista Eletrônica Extensão \& Sociedade, 2014; 5(2): 10-21.

17. LIBERA BD, BAIÃO MR, BARROS DC et al. Proposta de assistência nutricional pré-natal na atenção básica. Suplemento Revista Interface, 2014; 3(1):01

18. MENDONÇA FF, RETICENA KO. Perfil alimentar de gestantes atendidas em um hospital da região noroeste do Paraná. UNOPAR Científica, Ciências Biológicas e da Saúde, 2012; 14(2):99-104.

19. MOURA MDR, CASTRO MP, MARGOTTO PR et al. Hipertensão arterial na gestação - importância do seguimento materno no desfecho neonatal. Comunidade Ciências Saúde, 2011; 22(1):113-120.

20. ORGANIZACÃO MUNDIAL DE SAÚDE. Classificação do IMC em gestantes, 2003.

21. PADILHA PC, FREIRE K, SAUNDERS C. Associação entre o estado nutricional pré-gestacional e a predição do risco de intercorrências gestacionais. Revista Brasileira de Ginecologia e Obstetrícia, 2007; 29(10):511.

22. PACKER LW. Diabetes gestacional. Revista UNIPLAC, 2016; 4(1):04.

23. RAMOS GC, LAURENTINO AP, FOCHESATTO $S$ et al. Prevalência de infecção do trato urinário em gestantes de uma cidade do sul do Brasil. Revista Saúde Santa Maria, 2016; 42(1):173-178.

24. RIBEIRO AMC, SILVA CN, ROCHA GM et al. Pereira, Diabetes gestacional: determinação de fatores de risco para diabetes mellitus. Revista Portuguesa de Endocrinologia, Diabetes e metabolismo, 2015; 10(1):8-13

25. SANTOS KCR, MURARO LO, WITKOWSKI MC et al. Ganho de peso gestacional e estado nutricional do neonato: um estudo descritivo. Revista Gaúcha de Enfermagem, 2014; 35(1):62-69.

26. SOARES VMN, SOUZA KV, FREYGANG TC et al. Mortalidade materna por pré-eclâmpsia/eclampsia em um estado do Sul do Brasil. Revista Brasileira de Ginecologia, 2009; 31(11):566-573.

27. SOUSA DKS, BORGES NR, PEREIRA RJ et al. Influência dos desvios nutricionais gestacionais no peso ao nascer de recémnascidos atendidos pela rede pública de saúde do município de Palmas - TO. Revista CERUS, 2015; 7(1):111-126.

28. STULBACH, TE; BENÍCIO, MHD.; ANDREAZZA, $R$ et al. Determinantes do ganho ponderal excessivo durante a gestação em serviço público de pré-natal de baixo risco. Revista Brasileira de Epidemiologia, 2007; 10(1):99-108.

29. XAVIER RB, JANNOTTI CB, SILVA KS et al. Risco reprodutivo e renda familiar: análise do perfil de gestantes. Ciência \& Saúde Coletiva, 2013; 18(4):1161-1171. 